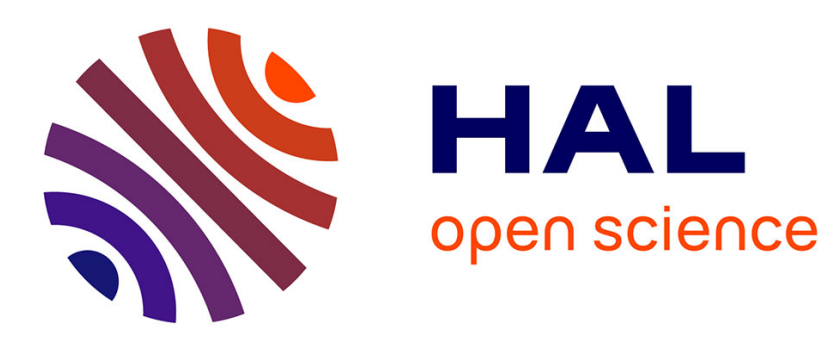

\title{
The Effect of Slice Orientation on Auditory fMRI at the Level of the Brainstem
}

Lavinia Slabu

\section{To cite this version:}

Lavinia Slabu. The Effect of Slice Orientation on Auditory fMRI at the Level of the Brainstem. Brain Topography: a Journal of Cerebral Function and Dynamics, 2010. hal-01865281

\section{HAL Id: hal-01865281 \\ https://hal.science/hal-01865281}

Submitted on 31 Aug 2018

HAL is a multi-disciplinary open access archive for the deposit and dissemination of scientific research documents, whether they are published or not. The documents may come from teaching and research institutions in France or abroad, or from public or private research centers.
L'archive ouverte pluridisciplinaire HAL, est destinée au dépôt et à la diffusion de documents scientifiques de niveau recherche, publiés ou non, émanant des établissements d'enseignement et de recherche français ou étrangers, des laboratoires publics ou privés. 


\title{
The Effect of Slice Orientation on Auditory fMRI at the Level of the
}

\section{Brainstem}

\author{
Lavinia M. Slabu ${ }^{1,2}$
}

${ }^{1}$ University of Barcelona, Department of Psychiatry \& Clinical Psychobiology, Institute for Brain, Cognition and Behavior (IR3C), Faculty of Psychology, P. Vall d'Hebron 171, 08035 Barcelona, Spain, e-mail: 1.m.slabu@ub.edu, Telephone: +34.93.312.50.66, Fax: +34 934021 584; ${ }^{2}$ University of Groningen, Neuroimaging Center, Graduate School of Behavioral and Cognitive Neuroscience, Groningen, The Netherlands 


\begin{abstract}
Although auditory information is processed in several subcortical nuclei, most fMRI studies focus solely on the auditory cortex and do not take brainstem responses into account. One common difficulty in obtaining clear functional brainstem recordings is due to heartbeat related motion, manifested in the rostro-caudal and in the ventro-dorsal directions in the contraction phase of the heart. The aim of this study was to investigate the effect of slice orientation on auditory functional magnetic resonance imagining (fMRI) measurements with respect to the pattern of brainstem oscillation. Fourteen healthy volunteers listened monaurally to modulated pink noise. Blood oxygenation level dependent (BOLD) contrast was performed with an echo-planar image (EPI) sequence using a 3 T MRI system. Three different slice orientations were compared: approximately parallel, at 45 degrees, and orthogonal to the brainstem. The standard deviation of the residuals, the effect size, the median $t$-values, and the number of activated voxels were calculated to quantify variability in activation between orientations. The data for the inferior colliculi indicated that a slice orientation with a 45 degrees angle to the brainstem yielded the lowest sensitivity to motion (reflected in the standard deviation of the residuals). By contrast, the results did not suggest differences between the three imaging planes on the scanning of the auditory cortex. Findings indicate that the 45 degrees slice orientation is the optimum orientation for accurate measurement at the upper brainstem level.
\end{abstract}

Keywords: inferior colliculus; slice orientation; brainstem motion; fMRI; 3T. 


\section{Introduction}

Movement is unavoidable in living human beings, and since the fMRI BOLD effects are relatively small (1-5\% signal changes), even a small amount of motion captured during an MRI can have an impact on acquisition (Cox and Jesmanowicz 1999) and can lead, for example, to image degradation and false-positive fMRI activation. In particular, functional imaging of the brainstem is complicated by heartbeat-related motion, blood flow, cerebrospinal fluid movement, tissue deformation, and the small size of the nuclei. While there is a large body of research dealing with activation of the auditory cortex (AC), limited information exists on functional imaging of the subcortical auditory pathway (Heßelmann et al. 2001). The subcortical areas involved in auditory information processing include: the cochlear nucleus $(\mathrm{CN})$, the superior olivary complex (SOC) at the lower brainstem level, the inferior colliculus (IC) in the upper brainstem, and the medial geniculate body (MGB) in the thalamus. The $\mathrm{CN}$ is located in the medulla and is approximately $2 \times 2 \times 5 \mathrm{~mm}$ (Hawley et al. 2005). The SOC is located in the pons and is approximately $3 \times 3 \times 7 \mathrm{~mm}$. In humans, the largest nucleus of the SOC, the medial superior olive, has a rostrocaudal extent of about 2.6 mm and a dorso-ventral extent of 1.8-2.4 mm (Bazwinsky et al. 2003; Marks et al. 1992). The ICs are the major site of converging projections from both lower and higher brain centres, and are supplied by blood vessels of different shapes and sizes, resulting in subject-specific motion. The size of the IC is approximately 6x6x4 mm (Hawley et al. 2005). This study addressed this gap in the literature by investigating the effect of slice orientation on auditory fMRI measurements with respect to the pattern of brainstem movement.

Motion of the brainstem arises from several correlated factors, such as vasculature (arteries and veins attached to the brainstem which cause a movement with each arterial pulsation), cerebrospinal fluid movement, and tissue deformation (Feinberg and Mark 1987). During the systolic (contraction) phase of the cardiac cycle, i.e. when the vessels pulsate, the brain tissue moves in the finite cranial volume. Several authors (Feinberg and Mark 1987; Enzmann and Pelc 1991; Poncelet et al. 1992; and Greitz et al. 1992) have described the motions of the brainstem in the rostro-caudal 
and in the ventro-dorsal directions in the contraction phase of the heart (cf. Figure $1 \mathrm{a}-\mathrm{c}$ ). However, the reported values for displacement and velocity in the caudal direction of the lower and upper brainstem differ considerably between authors (Feinberg and Mark 1987; Enzmann and Pelc 1991; Greitz et al. 1992; Maier et al. 1994). Generally, the central structures show caudal motion shortly after the systolic phase of the cardiac cycle. The caudal displacement occurs first in the cerebellar tonsil ( $69 \%$ of cardiac cycle), followed by displacement in the medulla, pons, midbrain and hypothalamus. The lower brainstem (medulla) has smaller amplitude in mean-displacement than the upper brainstem (midbrain) while the ventro-dorsal direction shows a similar cephalic progression of the motion from the medulla to the midbrain, but smaller in amplitude. The medulla $(\mathrm{CN})$ moves in directions opposite to those of the pons (SOC) and the midbrain (IC) late in the systolic phase of the cardiac cycle ( $94 \%$ of cycle). An additional and "somewhat surprising" difference according to Enzmann and Pelc's (1991) point of view is that cerebellar tonsils and lower brainstem, tractable by the spinal cord, have an earlier caudal motion than the upper brainstem (midbrain) and hypothalamus. In summary, the magnitudes of the lower brainstem and the upper brainstem motion reported in the literature appear to differ for the two directions due to the techniques used, or the group/individual analysis performed, covering the range from $0.11 \mathrm{~mm}$ with the cardiac cycle to approximately $2 \mathrm{~mm}$ with forced inspiration or breath-holding. 
Figure 1. The direction of forces responsible for brain motion (smaller arrows) and brainstem motion (larger arrows) in the sagittal (a), axial (b), and coronal (c) planes during systole (Greitz et al. 1992) and the movement of the brainstem in the rostro-caudal and in the ventro-dorsal planes (d).

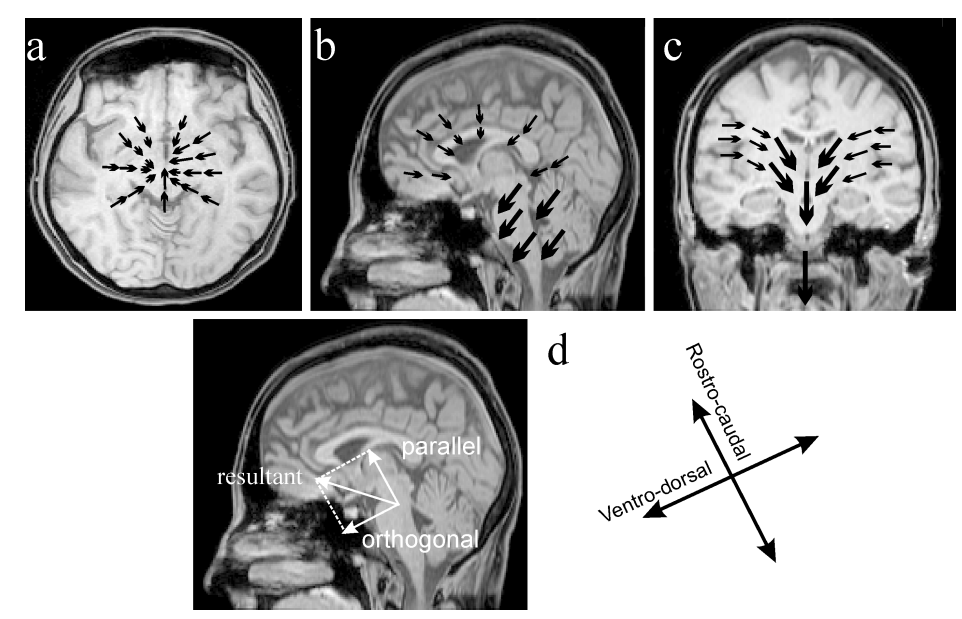

The variability observed in the literature regarding the source and the amplitude of the displacement between the upper and lower brainstem remains rather unclear, suggesting that further studies are needed to clarify this issue. Motion of cerebral cortex (including the AC) with the cardiac cycle is only 10-20\% of the brainstem motion (Enzmann and Pelc 1992; Greitz et al. 1992), approximately $0.05-0.1 \mathrm{~mm}$. Therefore, the present research focuses on the subcortical areas, where the motion represents a practical limit in imaging. Given the above arguments, it is expected that these differences in motion amplitude of the cortical and subcortical areas would be reflected in the fMRI data. Moreover, the present research assumes that the displacement of the brainstem in the cardiac cycle is $0.5 \mathrm{~mm}$ in $0.25 \mathrm{~s}$.

One method used in past research to compensate for brainstem motion is to use cardiac gating, such that image acquisitions are synchronized to the phase of the cardiac cycle (Vlaardingerbroeck and den Boer 1996). A pioneer study of auditory effects within the brainstem was conducted by Guimaraes and colleagues (1998) who used fMRI to demonstrate less signal variability when comparing cardiac gating to a non-gating condition in the inferior colliculi. 
Stronger signal intensity for the gated vs. the non-gated stimulus was shown at the auditory cortex. The authors report no significant difference of the BOLD response, only the standard deviation of the BOLD signal change was reduced at the inferior colliculi. Since their work, cardiac triggering has been frequently applied (Melcher et al. 2000; Griffiths et al. 2001; Harms and Melcher 2002; Backes and van Dijk 2002; Hawley et al. 2005; Sigalovsky and Melcher 2006; Schönwiesner et al. 2007). However, another line of research has performed scanning without cardiac gating, using different slice orientations (Heßelmann et al. 2001; Yetkin et al. 2004; Langers et al. 2005; Kovacs et al. 2006). For instance, Yetkin and colleagues (2004) and Langers and colleagues (2005) scanned a coronal plane orientation and they demonstrated consistent activation of the MGB and the IC and in a few cases activation of $\mathrm{CN}$, SOC and lateral lemniscus. Using an axial plane orientation, Heßelmann and colleagues (2001), observed activation of only the lower brainstem (i.e. CN and SOC).

The objective of this study was to investigate the effect of slice orientation on the measured activity at the level of the brainstem and auditory cortex, and ignore the possible effect of cardiac gating. Building on the results mentioned above (Heßelmann et al. 2001; Yetkin et al. 2004; Langers et al. 2005; Kovacs et al. 2006), it was assumed that the benefit of cardiac gating is negligible.

Motion of the brainstem consists in principle of an in-plane and a through-plane component. The relative relevance of these components depends on the direction of motion with respect to slice orientation. Furthermore, brainstem motion is described to have components in the rostro-caudal and ventro-dorsal directions, based on cine MRI (e.g., Poncelet et al. 1992; Greitz et al. 1992). If these components are in phase and of similar strength, which is our working hypothesis, then the resultant of these two components is at 45 degrees. Motion sensitivity is different between in-plane encoding (i.e. frequency and phase) and through-plane encoding (i.e. slice selection). Specifically, in-plane motion of spins (within gradient magnetic fields) modifies the phase of MR signals and shows the strongest effect if the motion is in the phase encoding direction, while through-plane 
motion of spins has an effect via the spin-history (Muresan et al. 2005). Unperturbed spins, typically inflowing blood or cerebrospinal fluid (CSF), result in increased signal intensity. Other spins may, due to the through-plane motion, experience multiple radio frequency pulses in rapid succession, causing saturation and thus a signal decrease. These effects will not be stable over multiple volume acquisitions due to the different phases of the heartbeat. The variance in measured signal intensity will be highest when motion is largest. Thus, an appropriate measure of motion sensitivity is the analysis of the residual motion, rather than the number of activated voxels, because of the possible false-positive fMRI activations that might occur at the brainstem level. In the present study, the fMRI data were acquired with an isotropic voxel size $\left(2 \times 2 \times 2 \mathrm{~mm}^{3}\right)$, and thus the main difference between orientations was the spatial encoding method for a given axis (i.e. frequencyencoding, phase-encoding, or slice selection).

Three different slice orientations perpendicular to the sagittal plane were used in the current experiment: orthogonal to the brainstem and to the rostro-caudal direction of motion of the brainstem - called orthogonal, parallel with the brainstem - named parallel, and at 45 degrees to the brainstem and to the already defined orientations - termed 45 degrees (see Figure 1d; Figure 2 a-c). The phase direction was always left-to-right. The through-plane motion of the brainstem was expected to dominate the measured signal and be least reflected in the 45 degrees orientation plane. A comparison with auditory cortex data was also performed, where according to the literature, the motion is small compared to the IC.

Figure 2. The imaging planes for the functional scan sessions: a) parallel, b) orthogonal, and c) at 45 degrees to the brainstem in the sagittal view, and d) fMRI design: the acoustic stimuli (black bars), were presented between two volume acquisitions (gray bars). 

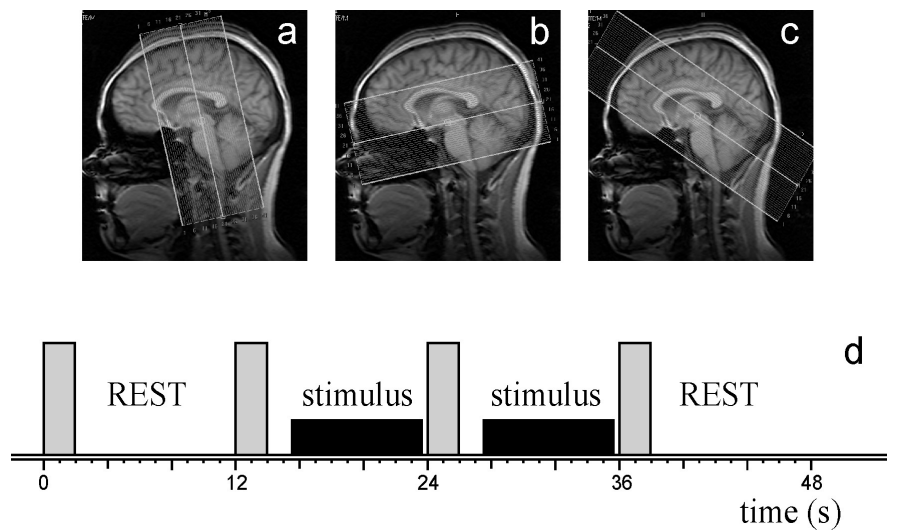

\section{Materials and methods}

\section{Subjects and task}

Fourteen subjects participated in this study that was approved by the Medical Ethics Committee of Groningen. All participants had normal hearing (hearing loss $<20 \mathrm{~dB}$ ). To minimize head movement during the scanning sessions, head padding and a strap were mounted on each side of the head coil and positioned on the subject's forehead. Datasets from four out of the 14 participants were excluded because of excessive sudden head motions (translation $>6 \mathrm{~mm}$; rotation $>4$ degrees), which occurred during the long scanning sessions (approximately 1.5 hours), either as a consequence of coughing or swallowing, or during a related response task. The mean and the standard deviation of absolute values of motion parameters of the remaining scans were 0.19 $\mathrm{mm} \pm 0.08 \mathrm{~mm}$ for the translation and $0.03^{\circ} \pm 0.001^{\circ}$ for the rotation. The data from ten participants (aged 23 to 33 years; 6 females; 1 left-handed) were analyzed. The light was dimmed during the experiments and the participants were blindfolded to minimize visual stimulation. The stimuli consisted of dynamic ripples generated by modulation of pink noise in the temporal and spectral domain. The stimuli were delivered separately to the left and right ear, with a duration of $8 \mathrm{~s}$. The ripples were characterized by four parameters: the spectral modulation density of 1 cycle per octave, the temporal modulation frequency of $8 \mathrm{~Hz}$, the modulation amplitude of $80 \%$, and an upward drift direction (cf. Langers et al. 2003; Langers et al. 2005). Stimuli were generated using a 
computer equipped with a digital-to-analog converter. The output of the converter was connected to the standard sound system of the MRI (Philips Medical Systems, Best, the Netherlands).

At the start of each experiment, the sound pressure level (SPL) was measured with the Brüel Kjær (Nærum, Denmark) artificial ear Type 4153. Stimuli were presented at $75 \mathrm{~dB}$ SPL, after a reduction of sound level (max. $31 \mathrm{~dB}$ ) by using earplugs. Due to technical modifications of the scanner, sound was delivered at $69 \mathrm{~dB}$ SPL for the last 6 participants. The participants had to report if the stimuli were perceived in the left or right ear, using an optically wired response box (FORP system, Current Designs Inc., Philadelphia, PA, USA). Stimuli were presented in pseudo random order and the numbers of right vs. left stimuli were balanced.

\section{fMRI design}

A sparse sampling fMRI design $(\mathrm{TR}=12 \mathrm{~s})$ was used, with a delay from the end of image acquisition until the beginning of the stimuli presentation of $1.5 \mathrm{~s}$. The paradigm contained two stimuli (both either left or right), followed by a silent baseline (REST) condition (cf. Hall et al. 1999), resulting in a series of 60 stimuli (30 right, 30 left), and 30 REST periods (Figure 2-d).

\section{Data acquisition}

Anatomical structures were identified using landmarks visible on the reference scan. The scanning planes intersected the CN, SOC, IC, MGB, and the AC. Images were collected using a $3 \mathrm{~T}$ Philips Intera scanner (Philips Medical Systems, Best, the Netherlands) equipped with a 6-channel SENSE head coil. Each session included three functional scans and 3 anatomical scans each containing 41 slices oriented parallel, orthogonal and at 45 degrees to the brainstem (Figure 2 a-c). The mean values and the standard deviation of the slice tilt relative to the AC-PC direction for the 10 subjects were $32^{0} \pm 2.77$ for the 45 degrees, $77.24^{0} \pm 3.04$ for the coronal, and $-11.85^{0} \pm 3.51$ for the orthogonal orientation planes. The IC having a size of approximately $6 \times 6 \times 4 \mathrm{~mm}^{3}$, usually covers 3 to 4 slices with the voxel size of $2 \times 2 \times 2 \mathrm{~mm}^{3}$. The functional scans consisted of $\mathrm{T}_{2}^{*}$ 
weighted EPI images (repetition time $\mathrm{TR}=12 \mathrm{~s}$, echo time $\mathrm{TE}=20 \mathrm{~ms}$, flip angle $90^{\circ}$, filed of view FOV $=192 \mathrm{~mm}$, matrix scan 96x96, SENSE factor $=2.3,41$ ascending slices, $2 \mathrm{~mm}$ slice thickness). The phase encoding was always in the left-to-right direction. The scan duration was 2015 ms per volume. Total number of volumes was 90. The scan parameters for the three anatomical images (gradient echo) were: $\mathrm{TR}=25 \mathrm{~ms}, \mathrm{TE}=20 \mathrm{~ms}$, flip angle $=30^{\circ}, \mathrm{FOV}=256$ $\mathrm{mm}$, matrix size 256x256, 41 slices, $2 \mathrm{~mm}$ thickness, no slice gap. The acquisition order of the 3 orientations was randomized over subjects in each imaging session.

\section{Data analysis}

Individual EPI time series were processed using the Statistical Parametric Mapping software ${ }^{\mathrm{i}}$. The preprocessing steps included: realignment, motion correction, and smoothing using an isotropic 3-mm Gaussian kernel. Data were not normalized. After applying the general linear model, the following contrasts were made: left stimuli vs. REST and right stimuli vs. REST. The tmaps were overlaid on top of the mean - realigned EPI images after applying a threshold of $\mathrm{t} \geq 3.21$ (corresponding to $\mathrm{p}<0.001$, uncorrected for multiple comparisons).

The regions of interest (ROI) were defined based on the activation clusters. Specific anatomical landmarks (Gebarski et al. 1993; Yetkin et al. 2004; Hawley et al. 2005; Sigalowsky and Melcher 2006) and sketches from Duvernoy (1995) were used to help identify the location of the activation clusters from the left and right ear stimulation for the left and right $\mathrm{CN}, \mathrm{SOC}, \mathrm{IC}, \mathrm{MGB}$, and AC. For greater statistical reliability, the data were analyzed separately for ipsilateral and contralateral sides, but pooled across ears of presentation.

The number of activated voxels (N), $t$-values, effect size (Es), and standard deviation (SD) of the residuals were analyzed to identify differences in scanning plane orientations. The residuals reflect the variance in the data that is not explained by the model and can reveal regions that are sensitive to the motion of the brain, respectively brainstem motion. The effect size is defined (in

\footnotetext{
${ }^{\text {i }}$ SPM99 (www.fil.ion.ucl.ac.uk/spm) was used because of the implemented MarsBaR toolbox (http://marsbar.sourceforge.ne) for defining the regions of interest.
} 
terms of SPM) as the components in vector $\beta$ in $\mathrm{Y}=\mathrm{X} \beta+\varepsilon$, where $\mathrm{Y}$ is a vector containing the measured signal, $\mathrm{X}$ is a vector containing the model, where each column contains a regressor, and $\varepsilon$ contains the error/residual. It is important to note that the values of $\beta$ are in arbitrary units. However, because the variance is equal for the different conditions in the design matrix, (both between and within subjects) these units are considered comparable (Friston et al. 1994).

To determine whether the distribution differs significantly between the orientations (for the sample size of $\mathrm{N}=10$ ), the two-sample Kolmogorov-Smirnov test (KS test) from SPSS 14.0 (SPSS Inc., Chicago, IL) was used. The KS test is a non-parametric test which has no restrictions for the underlying probability distribution(s). The KS test is not only sensitive to differences in the location of distributions, but is also greatly affected by differences in their shapes. Because the data were not normalized across slice orientations, the distributional comparison would preclude a significant outlier from generating a marked difference if the remainders of the data are consistent across two slice planes. In case there was no normal distribution, a Kruskal-Wallis test (the nonparametric analogue of the parametric analysis of variance ANOVA, except that it is based on ranks rather than means) was applied. After applying the Bonferroni correction, orientations were considered significant different if the probability fell below 0.016 . For a simple graphical representation of the distribution, the bar-graph of the mean, including the standard deviation is presented.

\section{Results}

\section{Brainstem}

Table 1 shows the detection level of the activation of the CN, SOC, IC, MGB and AC for the 10 subjects for three orientation planes. Because the MGB, CN and SOC were insufficiently activated across subjects, the analysis was focused on the IC and AC. The activation of the IC was almost always detected across subjects. Yet, one subject did not show significant activation for the 45 degrees orientation plane. The total number of activated voxels for the IC across subjects for the three orientation planes is presented in Table 2. Even though the mean values of the number of 
active voxels was higher for the contralateral than ipsilateral IC for the three slice orientations, a significant statistical difference was found only for the right stimulation (Kruskal-Wallis test, $\mathrm{p}=0.05)$. The comparison between the orientations revealed no significant difference for the range of the mean-corrected $t$-values and number of voxels at the IC for the ipsilateral or contralateral sides (Table 3). The $t$-values are proportional to Es divided by residual noise (SD). To study these parameters independently, the SD of the residuals and the Es were calculated (Table 3). The KS test showed significant effects on the SD (the top-right panel of Figure 3) between 45 degrees and orthogonal orientations, but not on the Es (the top-left panel of Figure 3). Moreover, the KruskalWallis test showed differences between the three orientation planes for the SD of the residuals in the IC controlateral to the attended ear $(p=0.0002)$, but not for the ipsilateral side $(p=0.067)$. Posthoc repeated measures contrasts confirmed differences between coronal and orthogonal $(p=0.016)$, 45 degrees and coronal $(p=0.017)$, and 45 degrees and orthogonal $(p=0.0002)$ orientation planes for the contralteral IC and between 45 degrees and orthogonal orientation planes for the ipsilateral IC to the attended ear $(p=0.005)$. 
Figure 3 The mean and standard deviation of the effect size, SD of the residuals, $t$-values, and number of activated voxels across subjects for the ipsilateral and contralateral sides pooled across ears of stimulation at the IC level. The significant (Kruskal-Wallis ANOVA test) differences of the parameters between orientations were noted with asterisks.
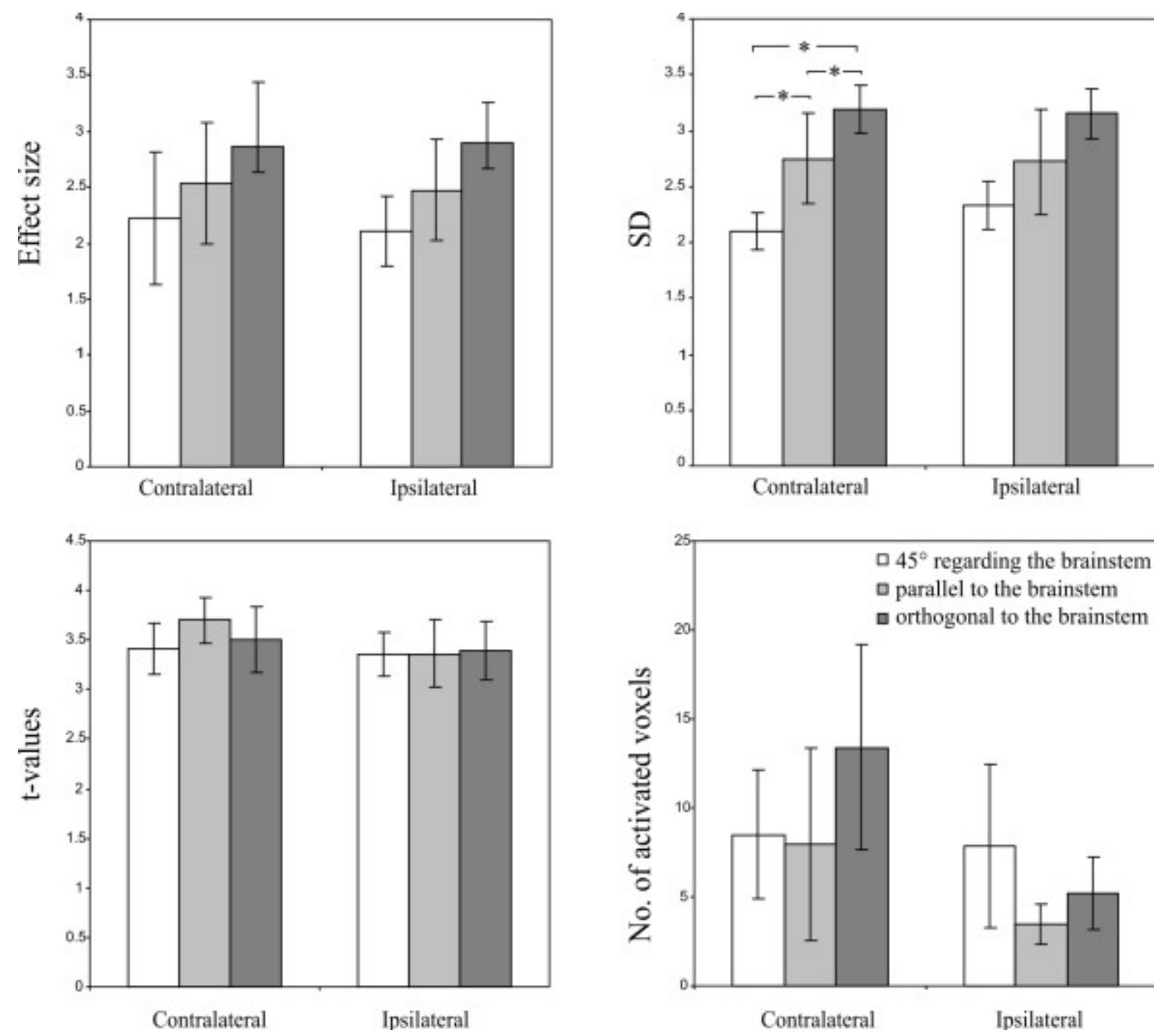

Table 1 . The number of subjects $(\max =10)$ showing activation at a threshold of $\mathrm{t} \geq 3.21$ (corresponding to $\mathrm{p}<0.001$, uncorrected for multiple comparisons).

\begin{tabular}{lccc}
\hline & \multicolumn{3}{c}{ paralle } \\
& 45 degrees & & orthogonal \\
\hline Cochlear Nucleus & 5 & 7 & 6 \\
Superior Olivary Complex & 5 & 5 & 6 \\
Inferior Colliculus & 9 & 10 & 10 \\
Medial Geniculate Body & 1 & 5 & 2 \\
Auditory Cortex & 10 & 10 & 10 \\
\hline
\end{tabular}


Table 2. The total number of activated voxels in the IC. The number of degrees of freedom is $72, \mathrm{t}$ $\geq 3.21$ (corresponding to $\mathrm{p}<0.001$ uncorrected for multiple comparisons).

\begin{tabular}{cccc}
\hline & 45 degrees & parallel & orthogonal \\
Subject & $N$ & $N$ & $N$ \\
\hline 1 & 12 & 23 & 19 \\
2 & - & 5 & 24 \\
3 & 11 & 41 & 24 \\
4 & 25 & 13 & 47 \\
5 & 1 & 14 & 2 \\
6 & 4 & 8 & 9 \\
7 & 35 & 1 & 19 \\
8 & 5 & 2 & 11 \\
9 & 13 & 6 & 6 \\
10 & 3 & 9 & 54 \\
Mean & 12.11 & 12.20 & 21.5 \\
SD & 11.26 & 12 & 17.06 \\
\hline
\end{tabular}

Table 3 The significance values of the Kolmogorov-Smirnov test for the SD, Es, $t$-values, and $\mathrm{N}$ for the ipsilateral and contralateral sides pooled across ears of presentation at the inferior colliculi.

\begin{tabular}{|c|c|c|c|c|c|c|}
\hline \multirow{5}{*}{ Parameters } & \multicolumn{3}{|c|}{ Contralateral } & \multicolumn{3}{|c|}{ Ipsilateral } \\
\hline & 45 & 45 degrees & parallel & 45 & 45 degrees & parallel vs. \\
\hline & degrees & vs. & $v s$. & degrees & $v s$. & orthogonal \\
\hline & $v s$. & orthogonal & orthogonal & $v s$. & orthogonal & \\
\hline & parallel & & & parallel & & \\
\hline$S D$ & 0.47 & $0.00^{*}$ & 0.04 & 0.34 & $0.00^{*}$ & 0.20 \\
\hline Effect size & 0.15 & 0.06 & 0.06 & 0.36 & 0.04 & 0.71 \\
\hline t-values & 0.07 & 0.68 & 0.46 & 0.61 & 0.52 & 0.99 \\
\hline$N$ & 0.29 & 0.51 & 0.13 & 0.90 & 0.85 & 0.66 \\
\hline
\end{tabular}

Visual inspection of the data revealed that the activation occurs also in the immediate vicinity of the colliculi using the scan plane orthogonal to the brainstem, and for the 45 degrees orientation plane the activation is lying directly on the IC (see Figure 4). In order to verify this observation, the anatomically-defined ROIs were analyzed. As depicted in Figure 4, the activation of the IC is displayed in one slice for the 45 degrees and orthogonal directions applying the comparison left stimuli vs. baseline (REST) for subjects 1, 2, 3, 5, and 6 or right stimuli vs. baseline 
(REST) for subjects 4, 7, 8, 9, and 10. For an easier visualization of the IC in the 45 degrees and orthogonal planes, the axial views are presented. The choice between left or right stimuli vs. baseline was based on the maximal activation of the orthogonal plane. The active areas outside of the IC are larger when the slices are positioned orthogonally to the brainstem compared with the 45 degrees orientation planes $(\mathrm{p}=0.03, \mathrm{KS}$ test $)$.

Fig. 4 fMRI activation maps of the IC in one slice applying the comparison left stimulus vs. baseline (REST) or right stimulus vs. baseline (REST), using the imaging plane orthogonal and at 45 degrees to the brainstem for the ten subjects (overlaid with mean EPI images, using a threshold for activation of $\mathrm{t} \geq 3.21$, corresponding to $\mathrm{p}<0.001$ uncorrected for multiple comparisons) for the axial view; left/right placement of the symbol represents left / right stimulation.

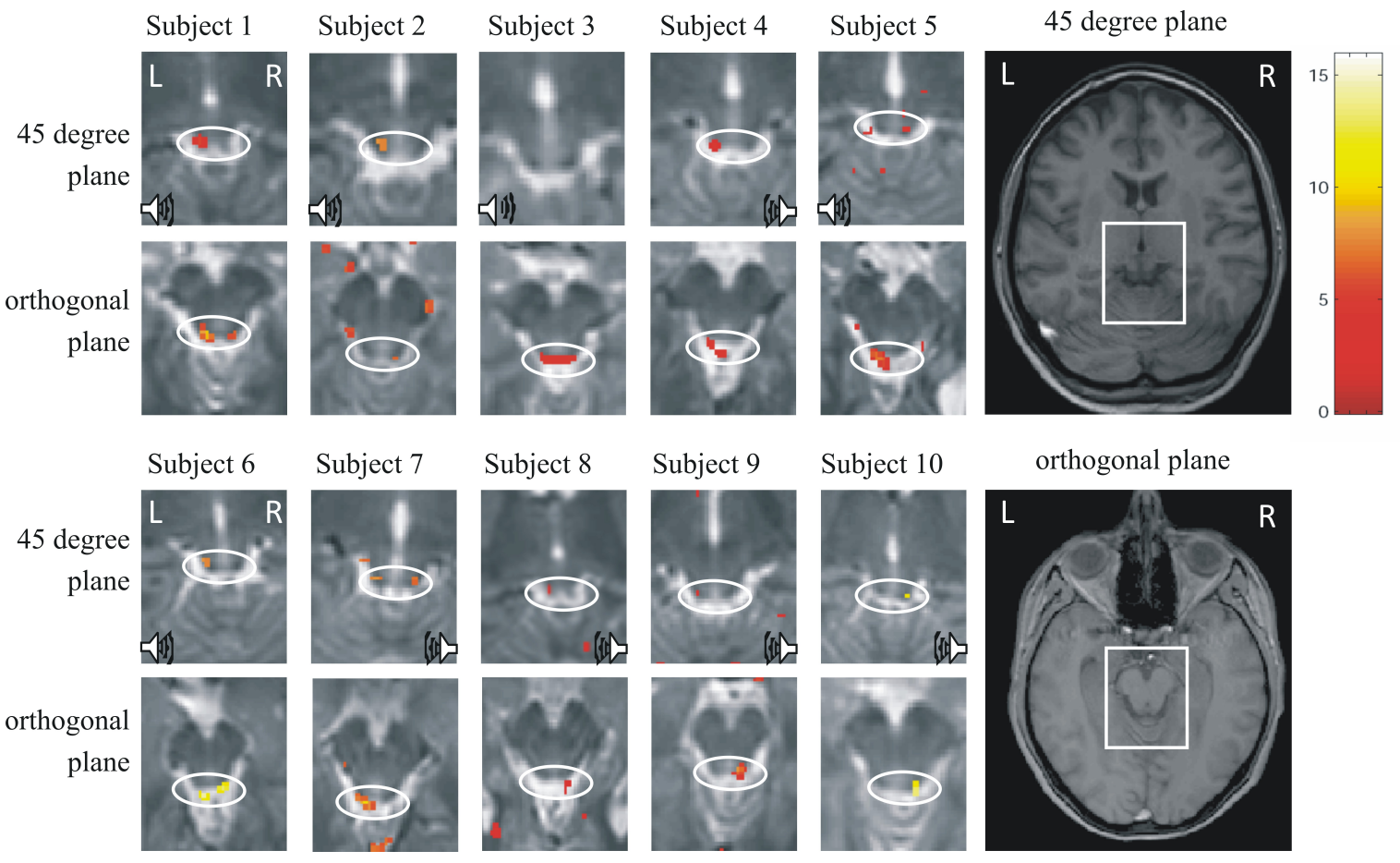




\section{Auditory cortex}

The same parameters were calculated for the AC. The KS test revealed no significant effects on the $\mathrm{SD}$, Es, mean-t values, and $\mathrm{N}$ with regard to the orientation plane in the AC (Figure 5).

Figure 5 The mean and standard deviation of the Es, SD of the residuals, $t$-values, and number of activated voxels across subjects for the ipsilateral and contralateral sides pooled across ears of stimulation at the AC level.
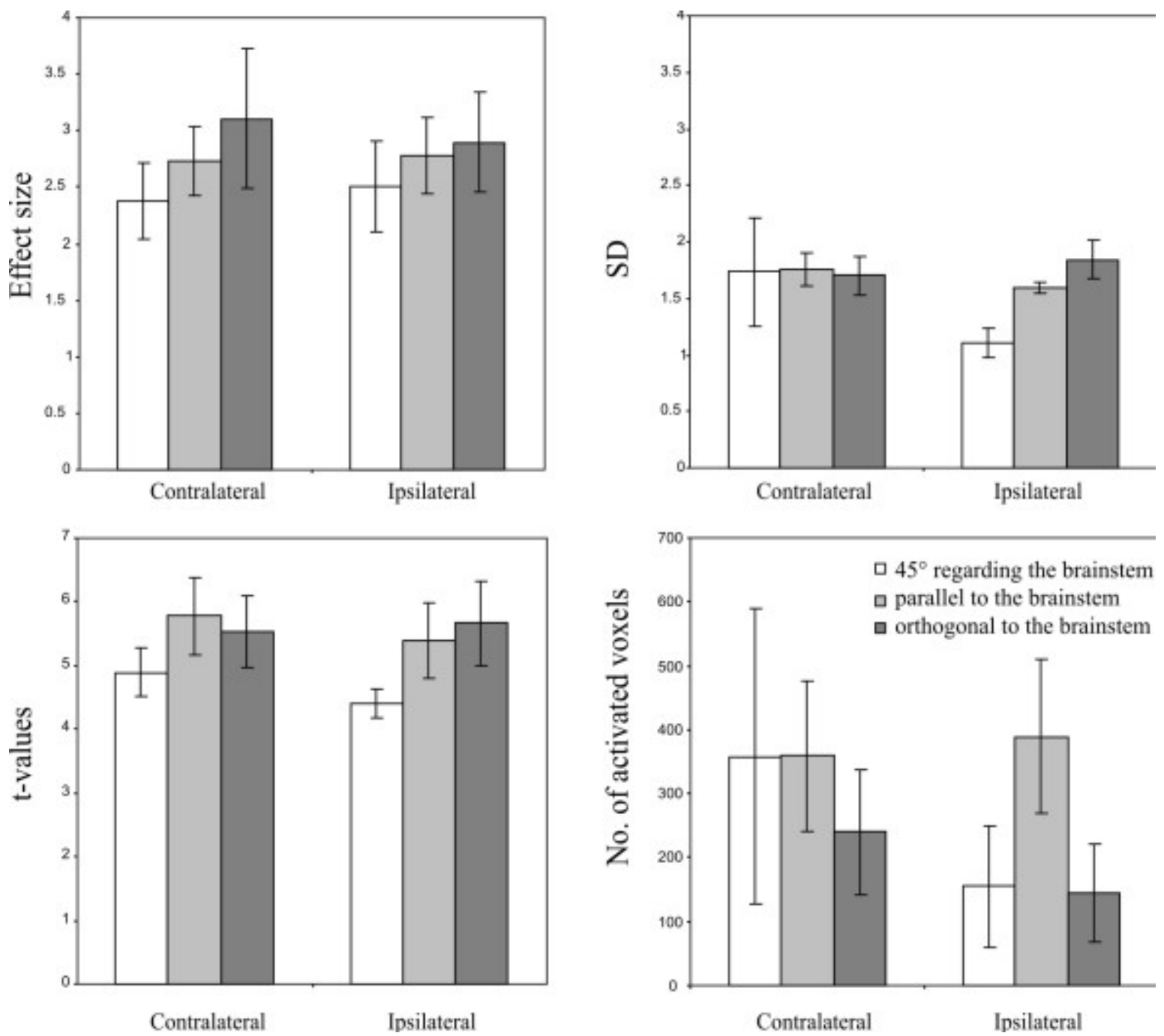


\section{Discussion}

\section{Brainstem}

The present findings are consistent with research by Langers and colleagues (2005) on a 1.5T scanner indicating a low detection level of the activation of the $\mathrm{CN}$, and SOC (see Table 1). Moreover, a similar paradigm was used for the current study, which involved rippled noise as a stimulus (Langers et al. 2003). Their results for the most significantly active voxels were: 1 out of 8 for the left SOC, 6/8 for the right SOC, 0/8 for left $\mathrm{CN}$, and 6/8 for the right $\mathrm{CN}$ (Langers et al. 2005). The weak responses in the CN, SOC and MGB may be caused by the type of the stimuli used. Furthermore, the size and morphology of these nuclei inhibit detection of their activation (Hawley et al. 2005). An alternative explanation would be related to the fact that the blood flow at this level differs from that at the inferior colliculi (IC). Measurements of the blood flow in the cat brain have revealed the highest level in the inferior colliculus $(1.8 \mathrm{cc} / \mathrm{gm} / \mathrm{min})$ compared with the blood flow of other auditory nuclei and auditory cortex (Landau et al. 1955). Zeller and colleagues (1997) found the highest capillary density in the inferior colliculus in laboratory rats.

However, past research has argued that the BOLD effect is sensitive to the effects of large draining veins when the acquisition plane is perpendicular to the local vessels which can have profound implications for spatial specificity. Given that, it is not possible to separate true tissue signal from the intra and extravascular contribution induced by nearby large draining vessels and therefore, the area that shows an increase in fMRI signal can be larger than the area of neuronal activation (Krings et al. 1999; Disbrow et al. 2000; van Gelderen et al. 2005). The venous relationships in the posterior incisural space, lying behind the midbrain, are the most complex in the cranium, orienting transversally or vertically to the brainstem (Ono et al. 1984). The present research argues that the macrovascular contribution appears to be strongest in case of an orthogonal orientation to the brainstem due to the activation outside of the colliculi (Figure 4). Even though the scan planes intersect the arteries and veins at different angles and blood vessels occupy different proportions of a given voxel that could explain the difference in intra-subject variability (Table 2), 
the mean and standard deviation of the number of activated voxels did not reveal inter-orientation differences at the IC (the right-bottom panel of Figure 3).

This study explored the possibility to reduce motion artifacts in auditory activation in the brainstem by optimizing the slice orientation. At the higher brainstem level (inferior colliculus) differences were observed for the explored directions. The results showed that the mean-t value and Es was not significantly affected by the orientation plane whereas SD of the residuals depended on the orientation planes, increasing from 45 degrees to parallel to orthogonal orientation plane for the contralateral side of the acousting stimulation as illustrated in the top-right panel of Figure 3. Due to the cross-over nature of the ascending projections, for a monaural sound, the greatest response will be on the contralateral side of the IC (Langers et al. 2005). Consequently, the most reliable indicator of the effect of slice orientation on activity would also be found on the contralateral side of the IC.

\section{Auditory cortex}

The KS test yielded no significant differences between orientations for the SD, Es, mean-t values, and $\mathrm{N}$ across the orientation planes for the AC (Figure 5). The fact that no effect of slice orientation in the residual signal fluctuations was observed for the SD in the AC, but a significant effect was visible in the IC may be due to the fact that auditory cortex motion is smaller than the motion of the brainstem over the cardiac cycle, and so a higher slice orientation impact was observed on the IC compared to the AC.

The most commonly used hardware, pulse sequence, and analysis techniques for a sparse sampling design were used in this research. Lately, auditory neuroimaging is becoming an increasingly important area and for this to be successful requires robust and accurate methods for imaging the subcortical auditory system. Consequently, the present results indicate that the orientation of image acquisition at the IC may provide more reliable measures by decreasing the residual signal fluctuations of the BOLD responses. The slice orientations reported in the literature for imaging the auditory nuclei are coronal (Yetkin et al. 2004; Langers et al. 2005; Lanting et al. 2008) and axial 
(Heßelmann et al. 2001) orientations. From a methodological perspective, an accurate slice orientation selection will be beneficial for the standardization of the fMRI acquisition parameters in scanning the IC. However, there are many open-questions that have not been addressed in the current research. For example, additional empirical work is needed to further explore whether the motion artifacts are limited by the gain of a high temporal resolution, for instance, when applying fast imaging sequences, allowing simultaneous acquisition of multiple slices, or acquiring a single slice rather than multi-slice. Nevertheless, a high spatial resolution is necessary for imaging the small auditory nuclei. Therefore, the choice in imaging should reflect a reasonable compromise between the voxel size and implicitly by the matrix size, the field of view, and the slice profile with respect to the structure of interest. Moreover, future research is also required to test whether cardiac gating can be omitted when the slice orientation is chosen to minimize the sensitivity to movement. A comparison of orientation effects on cardiac gating vs. non-gating at the brainstem level would provide more detailed information. It is worth mentioning that cardiac gating eliminates the effect of motion that is directly correlated to the cardiac rhythm by decreasing the variance at the inferior colliculus level, and not increasing the relative signal change within this same region (Guimaraes et al. 1998).

This study explored the potential impact of slice orientation on the inferior colliculi. Although $t$-values are not significantly different for the IC using different slice orientations, there is decrease of motion reflected in standard deviation of the residuals from the orthogonal slices to parallel and to 45 degrees orientation slices for the inferior colliculi. In summary, the 45 degrees slice orientation is the optimum orientation for a more accurate measurement at the upper brainstem level.

\section{Acknowledgements}

This manuscript is based on the doctoral dissertation of the author, under general supervision of Prof. Ir. Hendrikus Duifhuis, Dept. of Biomedical Engineering, Neuroimaging 
Center, University of Groningen, with additional assistance by Remco Renken, Ph.D., Esther Wiersinga-Post, Ph.D., and Hans Hoogduin, Ph.D., Neuroimaging Center, University of Groningen. The author wishes to thank Anita Kuiper from Neuroimaging Center for assistance in data acquisition.

\section{References}

Backes WH, van Dijk P (2002) Simultaneous sampling of event-related BOLD responses in auditory cortex and brainstem. Magn Reson Med. 47(1): 90-96.

Bazwinsky I, Hilbig H, Bidmon HJ, Rübsamen R (2003) Characterization of the human superior olivary complex by calcium binding proteins and neurofilament H (SMI-32). J Comp Neurol. 456(3): 292-303.

Cox RW, Jesmanowicz A (1999) Real-time 3D image registration for functional MRI. Magn Reson Med 42(6): 1014-1018.

Disbrow EA, Slutsky DA, Roberts TPL, Krubitzer LA (2000) Functional MRI at 1.5 tesla: A comparison of the blood oxygenation level-dependent signal and electrophysiology. PNAS 97(17): 9718-9723.

Duvernoy HM (1995) The Human Brain Stem and Cerebellum: Surface, Structure, vascularization, Three Dimensional sectional Anatomy, and MRI. Spinger-Verlag Wien, New York.

Enzmann DR, Pelc NJ (1991) Normal flow patterns of intracranial and spinal cerebrospinal fluid defined with phase-contrast cine MR imaging. Radiology 178(2): 467-474.

Enzmann DR, Pelc NJ (1992) Brain motion: measurement with phase-contrast MR imaging. Radiology 185(3): 653-660.

Feinberg DA, Mark AS (1987) Human brain motion and cerebrospinal fluid circulation demonstrated with MR velocity imaging. Radiology 163(3): 793-799.

Friston KJ, Holmes A, Worsley KJ, Poline J-B, Frith CD (1994) Statistical parametric maps in functional imaging: A general linear approach. Hum Brain Mapp. 2(4): 189-210. 
Gebarski SS, Tucci DL, Telian SA (1993) The cochlear nuclear complex: MR location and abnormalities. AJNR Am J Neuroradiol. 14(6): 1311-1318.

Greitz D, Wirestam R, Franck A, Nordell B, Thomsen C, Stahlberg F (1992) Pulsatile brain movement and associated hydrodynamics studied by magnetic resonance phase imaging. The Monro-Kellie doctrine revisited. Neuroradiology 34(5): 370-380.

Griffiths TD, Uppenkamp S, Johnsrude I, Josephs O, Patterson RD (2001) Encoding of the temporal regularity of sound in the human brainstem. Nat Neurosci. 4(6): 633-637.

Guimaraes AR, Melcher JR, Talavage TM, Baker JR, Ledden P, Rosen BR, Kiang NY, Fullerton BC, Weisskoff RM (1998) Imaging subcortical auditory activity in humans. Hum Brain Mapp. 6(1): 33-41.

Hall DA, Haggard MP, Akeroyd MA, Palmer AR, Summerfield AQ, Elliott MR, Gurney EM, Bowtell RW (1999) "Sparse" temporal sampling in auditory fMRI. Hum Brain Mapp. 7(3): 213-223.

Harms MP, Melcher JR (2002) Sound repetition rate in the human auditory pathway: representations in the waveshape and amplitude of fMRI activation. J Neurophysiol. 88(3): 1433-1450.

Hawley ML, Melcher JR, Fullerton BC (2005) Effects of sound bandwidth on fMRI activation in human auditory brainstem nuclei. Hear Res. 204(1-2): 101-110.

Heßelmann V, Wedekind C, Kugel H, Schulte O, Krug B, Klug N, Lackner KJ (2001) Functional magnetic resonance imaging of human pontine auditory pathway. Hear Res. 158(1-2): 160164.

Kovacs S, Peeters R, Smits M, De Ridder D, Van Hecke P, Sunaert S (2006) Activation of cortical and subcortical auditory structures at $3 \mathrm{~T}$ by means of a functional magnetic resonance imaging paradigm suitable for clinical use. Invest Radiol. 41(2): 87-96. 
Krings T, Erberich SG, Roessler F, Reul J, Thron A (1999) MR Blood Oxygenation LevelDependent Signal Differences in Parenchymal and Large Draining Vessels: Implications for Functional MR Imaging. AJNR 20 (11): 1907-1914.

Landau WM, Freygang WH, Rowland LP, Sokoloff L, Kety SS (1955) The local circulation of the living brain; values in the unanesthetized and anesthetized cat. Trans Am Neurol Assoc 80: 125-129.

Langers DR, Backes WH, van Dijk P (2003) Spectrotemporal features of the auditory cortex: the activation in response to dynamic ripples. Neuroimage 20(1): 265-275.

Langers DR, van Dijk P, Backes WH (2005) Lateralization, connectivity and plasticity in the human central auditory system. Neuroimage 28(2): 490-499.

Lanting CP, de Kleine E, Bartels H, van Dijk P (2008) Functional Imaging of unilateraltinnitus using fMRI. Acta Otolaryngol 128(4): 415-21.

Maier SE, Hardy CJ, Jolesz FA (1994) Brain and cerebrospinal fluid motion: real-time quantification with M-mode MR imaging. Radiology 193(2): 477-483.

Marks MP, Pelc NJ, Ross MR, Enzmann DR (1992) Determination of cerebral blood flow with a phase-contrast cine MR imaging technique: evaluation of normal subjects and patients with arteriovenous malformations. Radiology 182(2): 467-476.

Melcher JR, Sigalovsky IS, Guinan JJ,Jr, Levine RA (2000) Lateralized tinnitus studied with functional magnetic resonance imaging: abnormal inferior colliculus activation. $\mathrm{J}$ Neurophysiol. 83(2): 1058-1072.

Muresan L, Renken R, Roerdink JB, Duifhuis H (2005) Automated correction of spin-history related motion artefacts in fMRI: simulated and phantom data. IEEE Trans Biomed Eng. 52(8): 1450-1460.

Ono M, Ono M, Rhoton AL, Barry M (1984) Microsurgical anatomy of the region of the tentorial incisura. J Neurosurg. 60(2): 365-399 
Poncelet BP, Wedeen VJ, Weisskoff RM, Cohen MS (1992) Brain parenchyma motion: measurement with cine echo-planar MR imaging. Radiology 185(3): 645-651.

Schönwiesner M, Krumbholz K, Rübsamen R, Fink GR, von Cramon DY (2007) Hemispheric asymmetry for auditory processing in the human auditory brain stem, thalamus, and cortex. Cereb Cortex. 17(2): 492-499.

Sigalovsky IS, Melcher JR (2006) Effects of sound level on fMRI activation in human brainstem, thalamic and cortical centers. Hear Res. 215(1-2): 67-76.

van Gelderen P, Wu CWH, de Zwart JA, Cohen L, Hallett M, Duyn JH (2005) Resolution and Reproducibility of BOLD and Perfusion Functional MRI at 3.0 Tesla. Magn Reson Med. 54: $569-576$.

Vlaardingerbroeck MT, den Boer JA (1996) Magnetic resonance imaging. Springer, Berlin.

Yetkin FZ, Roland PS, Mendelsohn DB, Purdy PD (2004). Functional Magnetic Resonance Imaging of Activation in Subcortical Auditory Pathway. Laryngoscope 114: 96-101.

Zeller K, Rahner-Welsch S, Kuschinsky W (1997) Distribution of Glut1 glucose transporters in different brain structures compared to glucose utilization and capillary density of adult rat brains. J Cereb Blood Flow Metab. 17(2): 204-209. 